\title{
Longitudinal anterior-to-posterior shift of collateral channels in patients with moyamoya disease: an implication for its hemorrhagic onset
}

\author{
Shusuke Yamamoto, MD, Satoshi Hori, MD, PhD, Daina Kashiwazaki, MD, PhD, Naoki Akioka, MD, \\ Naoya Kuwayama, MD, PhD, and Satoshi Kuroda, MD, PhD
}

Department of Neurosurgery, Graduate School of Medicine and Pharmaceutical Science, University of Toyama, Japan

OBJECTIVE This study aimed to assess longitudinal changes in the collateral channels originating from the lenticulostriate artery (LSA), posterior communicating artery (PCoA), and anterior and posterior choroidal arteries (AChA and $P C h A$, respectively) during disease progression and/or aging. The impact of collateral channels on onset type was also examined.

METHODS This study included 71 involved hemispheres in 41 patients with moyamoya disease. The disease was categorized into 6 stages according to Suzuki's angiographic staging system. The degree of development of each moyamoya vessel was categorized into 3 grades.

RESULTS The LSA started to dilate in stage 2 , showed the most prominent development in stage 3 , and decreased in more advanced stages $(p<0.001)$. The AChA most notably developed in stage 3 and gradually shrank $(p=0.04)$. The PCoA started to dilate in stage 3 and showed the most prominent development in stage $4(p=0.03)$. The PChA started to dilate in stage 3 and showed the most prominent development in stages 4 to $5(p<0.001)$. Patient age was negatively related to $L S A$ development $(p=0.01, R=0.30)$ and was positively associated with the abnormal dilation and extension of the PCoA $(p=0.02, R=0.28)$ and PChA $(p<0.001, R=0.45)$. The PCoA, AChA, and PChA more distinctly developed in hemispheres with intracerebral or intraventricular hemorrhage than in hemispheres with ischemic stroke or transient ischemic attack ( $p<0.001, p=0.03$, and $p=0.03$, respectively).

CONCLUSIONS This study suggests that the collateral channels through moyamoya vessels longitudinally shift from the anterior to posterior component during disease progression and aging, which may be closely related to the onset of hemorrhagic stroke in adult moyamoya disease.

https://thejns.org/doi/abs/10.3171/2017.9.JNS172231

KEY WORDS moyamoya vessel; perforating artery; cerebral angiography; Suzuki's angiographic staging; vascular disorders

$\mathrm{M}$ OYAMOYa disease (MMD) is a unique cerebrovascular disorder characterized by progressive occlusion of the terminal portion of the internal carotid artery and its main branches and the anterior and middle cerebral arteries. Typically, the perforating arteries, such as the lenticulostriate arteries (LSAs), are markedly dilated to supply collateral blood flow to the ischemic brain and are called moyamoya vessels. ${ }^{10}$ The posterior communicating artery (PCoA) and the anterior and posterior choroidal arteries (AChA and PChA, respectively) also serve the dilated perforating arteries and contribute as alternative collateral channels in patients with MMD. ${ }^{8}$ Therefore, moyamoya vessels have different origins in each involved hemisphere. Suzuki and Takaku categorized MMD into 6 stages on cerebral angiography. ${ }^{10}$ It is well known that moyamoya vessels originating from the LSAs are the most prominent in stage 3 and gradually diminish in more advanced stages. To the best of our knowledge, however, there are no studies that denote the longitudinal changes in moyamoya vessels originating from other arteries, such as the PCoA, AChA, and PChA.

On the other hand, MMD characteristically leads to di-

ABBREVIATIONS $A C h A=$ anterior choroidal artery; $L S A=$ lenticulostriate artery; $M M D=$ moyamoya disease $; \mathrm{PChA}=$ posterior choroidal artery; $\mathrm{PCOA}=$ posterior communicating artery; $\mathrm{TIA}=$ transient ischemic attack.

SUBMITTED September 6, 2017. ACCEPTED September 25, 2017

INCLUDE WHEN CITING Published online March 23, 2018; DOI: 10.3171/2017.9.JNS172231. 
verse symptoms, such as transient ischemic attack (TIA), ischemic stroke, and hemorrhagic stroke. Most pediatric patients develop TIA or ischemic stroke, while about half of adult patients experience hemorrhagic stroke., ${ }^{1,6,10}$ Although the cause of this discrepancy between pediatric and adult patients is still unclear, some studies have suggested that "specific" moyamoya vessels may play an important role in the occurrence of hemorrhagic stroke in adult patients with MMD. ${ }^{2,3,7,8}$ However, a recent clinical trial showed that direct or combined surgical revascularization significantly reduces the incidence of recurrent hemorrhagic stroke, probably by diminishing dilated, fragile moyamoya vessels. ${ }^{11}$ However, there are no radiological indicators that might be able to efficiently predict the occurrence of hemorrhagic stroke in the future.

Based on these observations, this study aimed to precisely assess longitudinal changes in collateral channels arising from the LSA, PCoA, AChA, and PChA during disease progression and/or aging. Furthermore, the impact of collateral channels on onset type was also examined.

\section{Methods \\ Patients}

This prospective study included a total of 41 patients who were admitted to our hospital due to MMD. All patients were diagnosed with MMD according to the guideline for the diagnosis of MMD set by the Research Committee on Moyamoya Disease of the Ministry of Health, Welfare, and Labor of Japan. ${ }^{9}$ There were 18 children and 23 adult patients (20 males and 21 females). The mean ages $( \pm \mathrm{SD})$ were $10.2 \pm 4.7$ years and $41.0 \pm 9.0$ years for the pediatric and adult patients, respectively. Among the 18 pediatric patients, 14 patients $(77.8 \%)$ had bilateral MMD and $4(22.2 \%)$ had unilateral MMD. Likewise, among the 23 adult patients, 16 patients $(69.6 \%)$ had bilateral MMD and $7(30.4 \%)$ had unilateral MMD. Taken together, 30 patients $(73.2 \%)$ were diagnosed with bilateral-type MMD and the remaining 11 patients $(26.8 \%)$ were diagnosed with unilateral-type MMD. As a result, 71 hemispheres in 41 patients were diagnosed as the involved sides. Among the pediatric patients, the clinical diagnosis included TIA in 12 patients (66.7\%), ischemic stroke in 5 (27.8\%), and asymptomatic presentation in $1(5.6 \%)$. Among the adult patients, TIA was diagnosed in 7 patients $(30.4 \%)$, ischemic stroke in $5(21.7 \%)$, and intracranial hemorrhage in 6 (26.1\%); 5 patients $(21.7 \%)$ were asymptomatic at presentation. This study was approved by the institutional review board at our hospital, and informed consent was obtained from each patient, with parents giving consent for the pediatric patients.

\section{Radiological Examinations}

On cerebral angiography, the disease stage was precisely determined in all 71 involved hemispheres of 41 patients with MMD. The disease was categorized into 6 stages according to Suzuki's angiographic staging system. ${ }^{10}$ Simultaneously, the development of moyamoya vessels was also precisely analyzed on cerebral angiography. Moyamoya vessels are dilated perforating arteries that originate from the LSA, AChA, PCoA, and PChA. In this

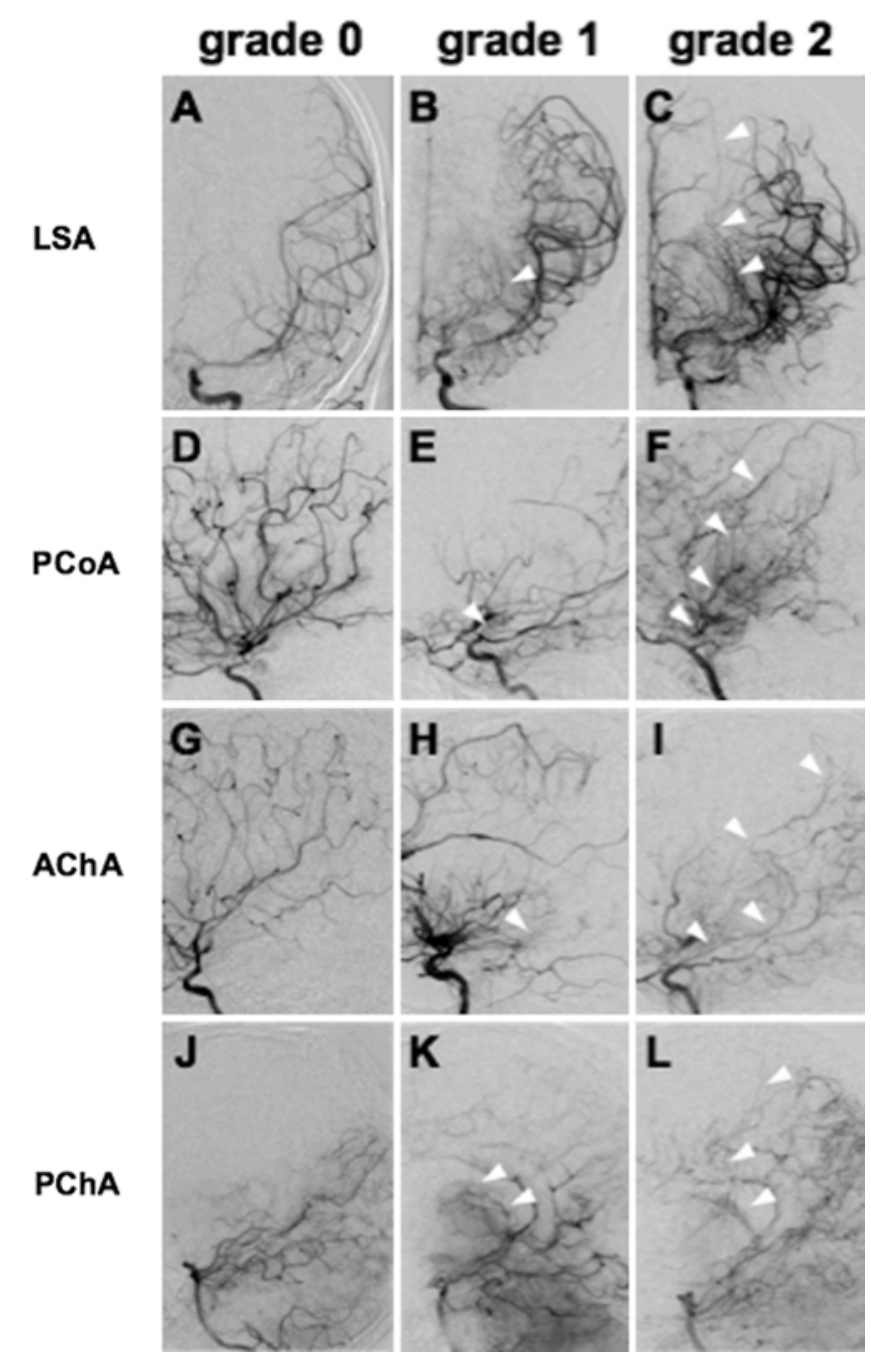

FIG. 1. Representative findings of the LSA, PCoA, AChA, and PChA on cerebral angiography. They were categorized into 3 grades according to the method of Morioka et al.

study, the degree of development of each moyamoya vessel was categorized into 3 grades according to the method of Morioka et al., ${ }^{8}$ with minor modifications: grade 0 , the perforating arteries do not dilate; grade 1, the perforating arteries increase in caliber but provide blood flow to only their original territory; and grade 2, the perforating arteries abnormally increase in caliber and start to provide collateral blood flow to the regions beyond their original territory. Typical findings of each grade are shown in Fig. 1. The angiographic findings were assessed by one of the authors (S.Y.). In this study, the degree of abnormal dilation and extension of each perforating artery was statistically analyzed in relation to disease stage, patient age, and onset type.

\section{Statistical Analysis}

Continuous data are expressed as mean \pm SD. Categorical variables were compared between groups using the chi-square test or Mann-Whitney U-test as appropriate. 


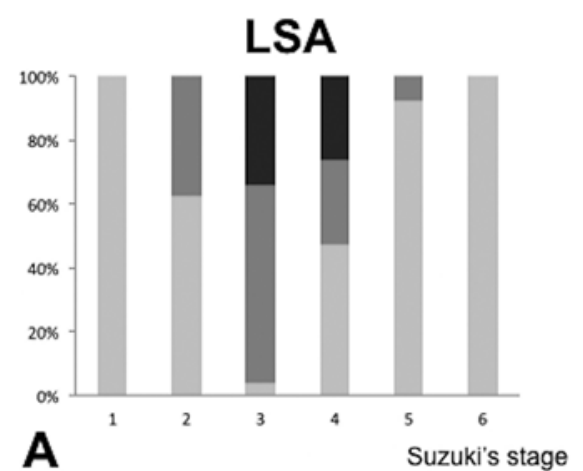

AChA

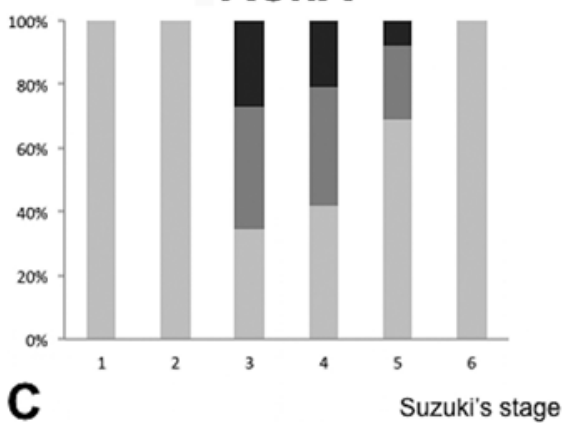

PCoA

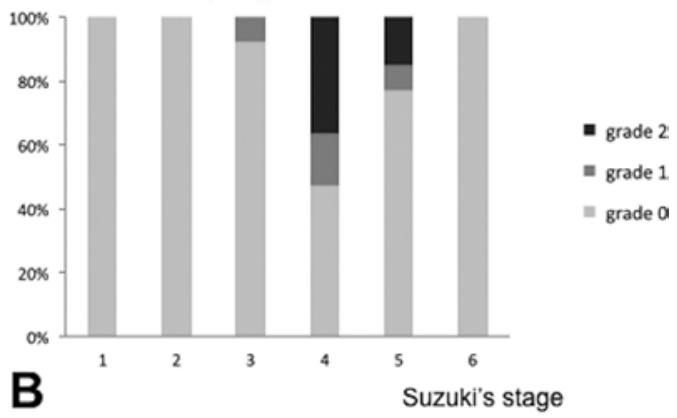

PChA

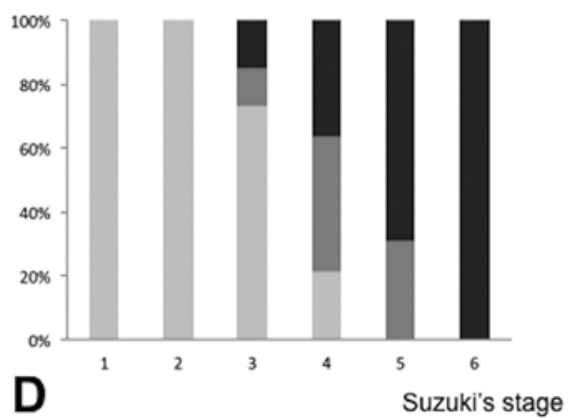

FIG. 2. Bar graphs demonstrating the relationship between Suzuki's angiographic stage and the abnormal dilation and extension of the LSA, PCoA, AChA, and PChA. A: The LSA starts to dilate in stage 2, shows the most prominent development in stage 3 , and gradually loses its role as the collateral channel and decreases in more advanced stages ( $p<0.001$, chi-square test). B: The PCoA starts to dilate in stage 3 , shows the most prominent development in stage 4 , and gradually loses its role as the collateral channel and decreases in more advanced stages $(p=0.04)$. C: The AChA starts to dilate in stage 3 , shows the most prominent development in stage 4 , and gradually loses its role as the collateral channel and decreases in more advanced stages $(p=$ 0.03). D: The PChA starts to dilate in stage 3 and shows the most prominent development in stages 4 to $5(p<0.001)$.

Differences were considered statistically significant at $\mathrm{p}$ $<0.05$.

\section{Results}

\section{Radiological Findings}

According to Suzuki's classification system, the 71 involved hemispheres were categorized into 6 stages: stage $1(n=4)$, stage $2(n=8)$, stage $3(n=26)$, stage $4(n=19)$, stage $5(n=13)$, and stage $6(n=1)$.

On cerebral angiography, the development of the moyamoya vessels in each perforating artery, including the LSA, AChA, PCoA, and PChA, was categorized into 3 grades. As a result, the LSA was classified as grades 0, 1 , and 2 in 32, 25, and 14 cases, respectively. The AChA was classified as grades 0,1 , and 2 in 39,20, and 12 cases, respectively. The PCoA was classified as grades 0,1 , and 2 in 56, 6, and 9 cases, respectively. Finally, the PChA was classified as grades 0,1 , and 2 in 35, 15, and 21 cases, respectively.

\section{Association Between Suzuki Angiographic Stage and Abnormal Dilation and Extension of the Perforating Arteries}

As the next step, the relationship between Suzuki's angiographic stage and the abnormal dilation and exten- sion of each perforating artery was analyzed. The results are shown in Fig. 2. The LSA started to dilate in stage 2 , showed the most prominent development in stage 3, and lost its role as a collateral channel in more advanced stages ( $p<0.001$, chi-square test). The AChA showed a very similar finding $(\mathrm{p}=0.04)$. The PCoA started to dilate in stage 3 , showed the most prominent development in stage 4, and lost its role as a collateral channel in more advanced stages $(p=0.03)$. On the other hand, the PChA showed a different pattern from that of the other perforating arteries. Thus, the PChA started to dilate in stage 3 and showed the most prominent development in stages 4 to 5 ( $p<0.001)$. Therefore, as disease stage advances, the moyamoya vessels originating from the vertebrobasilar system play a more important role as collateral pathways than those originating from the carotid system.

\section{Association Between Patient Age and the Development of Moyamoya Vessels in the Perforating Artery}

The relationship between patient age and the abnormal dilation and extension of each perforating artery was also evaluated. The results are summarized in Fig. 3. There was a significant negative correlation between the patient age and the abnormal dilation and extension of the LSA ( $p$ $=0.01, \mathrm{R}=0.30$ ). The abnormal dilation and extension of the AChA did not correlate with patient age $(p=0.74, R$ 

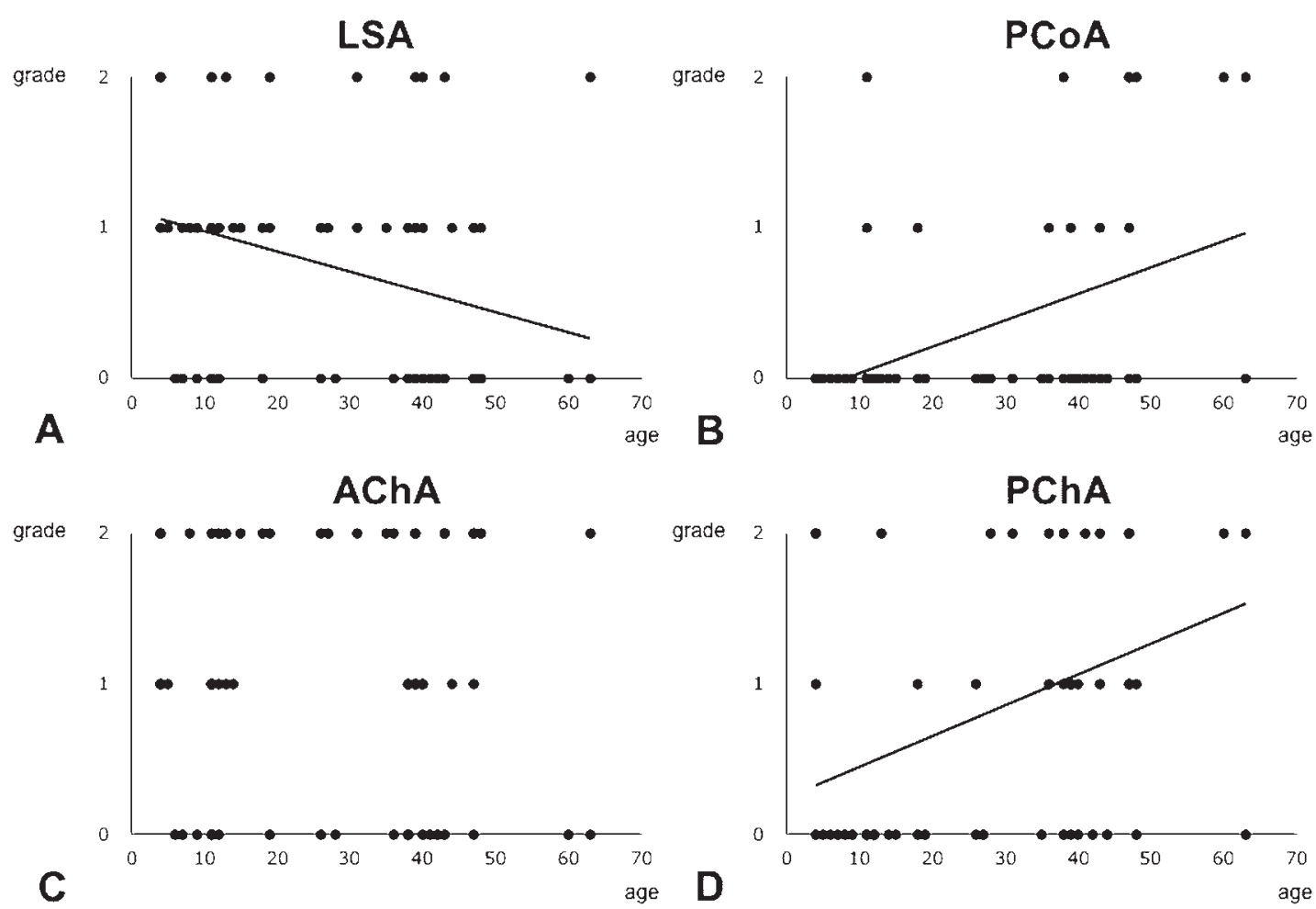

FIG. 3. Scatterplots demonstrating the relationship between patient age and the abnormal dilation and extension of the LSA, PCoA, AChA, and PChA. A: There is a significant negative correlation between patient age and the abnormal dilation and extension of the LSA ( $p=0.01, R=0.30)$. B and $D$ : There are significant positive correlations between patient age and the abnormal dilation and extension of the PCoA $(B ; p<0.001, R=0.43)$ and PChA $(D ; p<0.001, R=0.40)$. C: The abnormal dilation and extension of the AChA does not correlate with patient age $(p=0.74, R=0.04)$. Each dot represents a patient.

$=0.04)$. On the other hand, there were significant positive correlations between patient age and the abnormal dilation and extension of the PCoA $(\mathrm{p}<0.001, \mathrm{R}=0.43)$ and $\mathrm{PChA}$ $(\mathrm{p}<0.001, \mathrm{R}=0.40)$.

When the patients were categorized into 2 groups, the pediatric and adult groups, the finding was more notable (Fig. 3). Thus, the abnormal dilation and extension of the LSA was more remarkable in the pediatric group than in the adult group $(\mathrm{p}=0.01)$. There was no significant difference in the abnormal dilation and extension of the AChA between the 2 groups $(p=0.53)$. On the other hand, the abnormal dilation and extension of the PCoA and PChA was more prominent in the adult group than in the pediatric group ( $p=0.02$ and $p<0.001$, respectively). Therefore, as patient age advances, the moyamoya vessels through the PCoA and PChA play more important roles than those through the LSA.

\section{Association Between Onset Type and the Development of Moyamoya Vessels in the Perforating Artery}

To examine the relationship between onset type and the abnormal dilation and extension of each perforating artery, the 71 involved hemispheres were divided into 3 groups: ischemic $(n=51)$, hemorrhagic $(n=11)$, and asymptomatic $(n=9)$ hemispheres. In this study, asymptomatic hemispheres were excluded because it was unclear whether they would develop ischemic or hemorrhagic events in the future. The results are shown in Fig. 4. As a result, there was no significant difference between onset type and the abnormal dilation and extension of the LSA $(\mathrm{p}=0.33)$. On the other hand, the abnormal dilation and extension of the PCoA, AChA, and PChA was significantly more distinct in hemispheres with intracerebral or intraventricular hemorrhage (hemorrhagic-type hemispheres) than in hemispheres with ischemic stroke or TIA (ischemic-type hemispheres) ( $p<0.001, p=0.03$, and $p=0.03$, respectively; Mann-Whitney U-test).

\section{Discussion}

In this study, the first aim was to clarify the relationship between longitudinal changes in collateral channels and disease progression in patients with MMD. Based on the findings on cerebral angiography, this study clearly demonstrates that the collateral channels through the moyamoya vessels dramatically change as disease stage progresses. Although the dilation and extension of the LSA and AChA is remarkable in stage 3 hemispheres, they gradually decreased in caliber as the disease stage further progressed. Instead, the abnormal dilation and extension of the PCoA was most notable in the stage 4 hemispheres. On the other hand, the PChA started to increase in caliber and the findings became most prominent in stage 5 hemispheres, which was associated with disappearance of the PCoA. These results clearly suggest that the collateral 
LSA

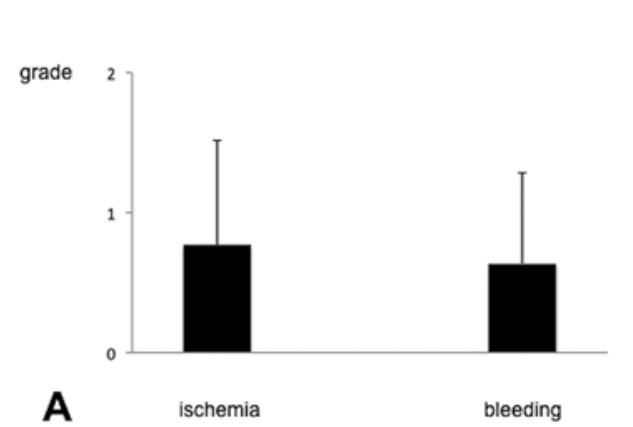

AChA

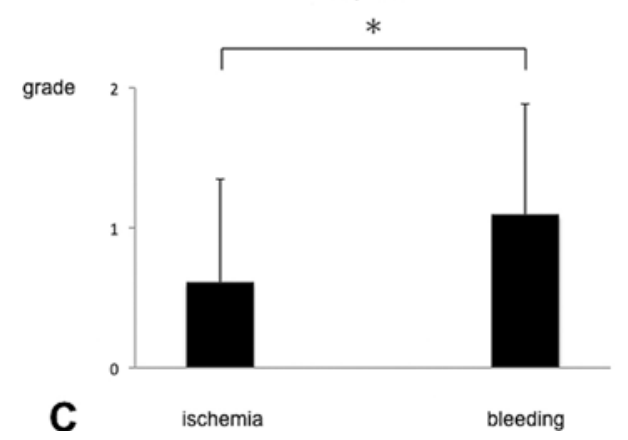

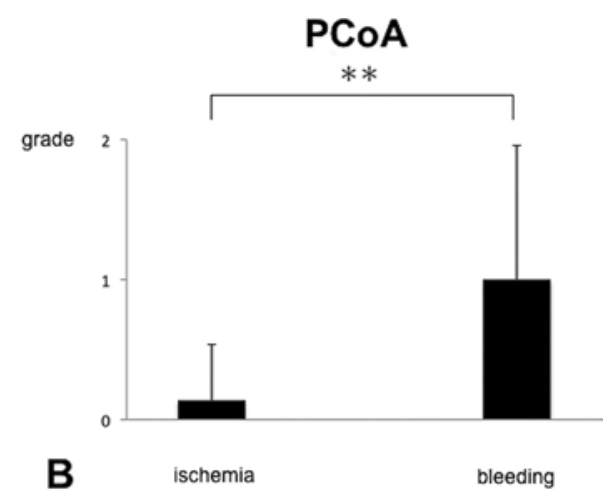

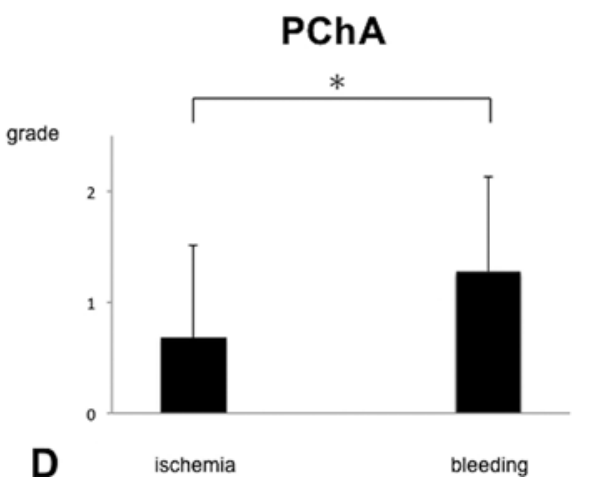

FIG. 4. Bar graphs showing the relationship between onset type and the abnormal dilation and extension of the LSA, PCOA, AChA, and PChA. A: There is no significant difference between onset type and the abnormal dilation and extension of the LSA $(p=0.33$, Mann-Whitney U-test). B-D: There are significant correlations between onset type and the abnormal dilation and extension of the PCoA (B; $p<0.001), \operatorname{AChA}(C ; p=0.03)$, and PChA (D; $p=0.03)$. ${ }^{*} p<0.05 ;{ }^{* *} p<0.01$.

channels through the dilated perforating arteries, i.e., the moyamoya vessels, shift from the anterior to the posterior segment in a stepwise manner as disease stage progresses (Fig. 5). There are no prior reports that evaluated changes in collateral channels through the progression of disease stage in MMD. Therefore, this is the first report that definitely demonstrates a close relationship between the "rise and fall" of moyamoya vessels and disease progression. We would like to propose that the longitudinal changes in moyamoya vessels should be called an "anterior-to-posterior shift" in patients with MMD; this is a novel concept that the role of the collateral channels gradually shifts from the LSA to the AChA and PCoA (carotid system) and finally to the PChA (vertebrobasilar system). Very recently, Funaki et al. proposed a novel concept about dangerous periventricular anastomosis in MMD., ${ }^{2,4}$ According to their findings on flow-sensitive black-blood MR angiography, the perforating arteries, such as the LSA, thalamic perforating arteries, and choroidal arteries, often created anastomoses with the medullary or insular arteries in the periventricular area to provide collateral blood flow to the cortex. Furthermore, they clearly showed that periventricular anastomosis is dangerously related to the onset of intracerebral hemorrhage in MMD. In the present study, the abnormal dilation and extension of the LSA, AChA, PCoA, and PChA was graded according to the method described by Morioka et al. ${ }^{8}$ Grade 2 abnormal dilation and extension of these arteries would represent a very similar finding to the periventricular anastomosis described by Funaki et al. ${ }^{4}$

Second, this study also examined the relationship between longitudinal changes in collateral channels and patient age. As a result, the role of the LSA as a collateral pathway gradually decreased as the patients became older, while the roles of the PCoA and PChA gradually increased in older patients. These results strongly suggest that the collateral channels through the moyamoya vessels also shift from the anterior (carotid) to posterior (vertebrobasilar) system in response to the patient aging. Furthermore, when the patients were categorized into pediatric and adult groups, there were significant differences in the development of the collateral channels between the groups. Thus, the abnormal dilation and extension of LSA was more remarkable in the pediatric group than in the adult group, and the abnormal dilation and extension of the PCoA and PChA was more prominent in the adult group than in the pediatric group. As reported by Morioka et al., the distinct abnormal dilation and extension of the PCoA and PChA is a predictor of subsequent hemorrhagic stroke. ${ }^{8}$ Therefore, this anterior-to-posterior shift in collateral channels may easily explain why adult patients are more prone to hemorrhagic stroke than pediatric patients.

Third, based on these observations, this study clearly demonstrates that the PCoA, AChA, and PChA show significantly more distinct abnormal dilation and extension in hemorrhagic-type hemispheres than in ischemic-type 

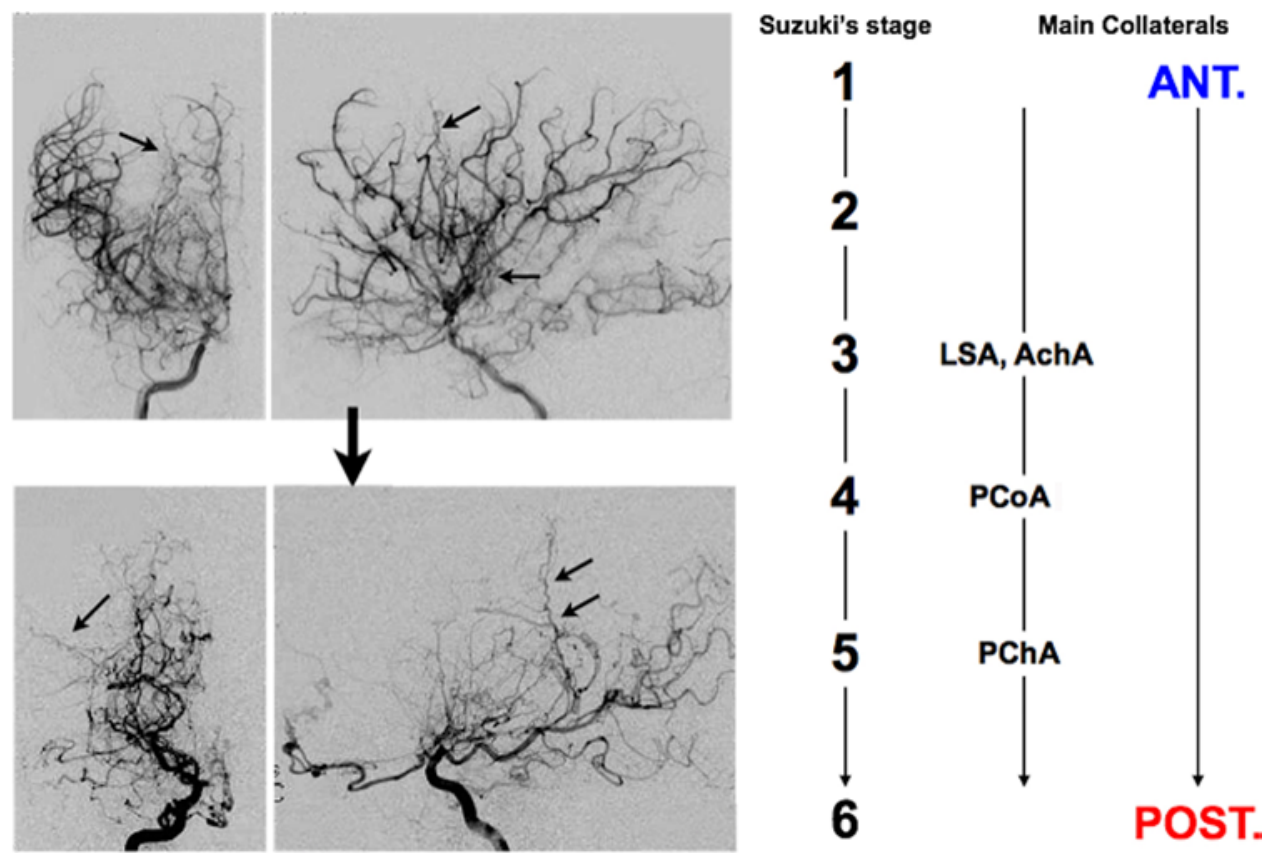

FIG. 5. Diagram showing the novel concept of the longitudinal shift of collateral channels through the moyamoya vessels from the anterior to posterior component during disease progression and aging, i.e., the anterior-to-posterior shift. Upper: Representative cerebral angiograms obtained in a 6-year-old girl who developed ischemic stroke. The LSA shows a marked dilation and extension to the frontal lobe (arrows, stage 3). Lower: Representative cerebral angiograms obtained in a 34-year-old woman who had a hemorrhagic stroke. The LSA does not play a major role as a collateral channel, while the PChA gives rise to a prominent collateral pathway to the parietal lobe (arrows, stage 5). Ant. = anterior; post. = posterior. Figure is available in color online only.

hemispheres. The finding is supported by previous reports. Irikura et al. reviewed the findings on cerebral angiography in 19 patients, including patients with hemorrhagictype $(\mathrm{n}=10)$ and ischemic-type $(\mathrm{n}=9)$ hemispheres, and found that a marked enlargement of the choroidal arteries was more remarkable in the hemorrhagic group than in the ischemic group. ${ }^{5}$ Liu et al. also evaluated the findings on cerebral angiography in 143 hemispheres (79 ischemic and 64 hemorrhagic hemispheres) and reported that the extension and excessive dilation of the AChA and PCoA was significantly correlated with the occurrence of hemorrhagic events. ${ }^{7}$ Furthermore, as shown in the previous report by Morioka et al., ${ }^{8}$ dilated and extended choroidal or thalamic perforating arteries may be more responsible for intracranial bleeding than the LSAs, which had traditionally been denoted as moyamoya vessels and suspected as a source of bleeding. Although these previous reports did not assess the PChA, Funaki et al. ${ }^{3}$ very recently showed that choroidal anastomosis is an independent factor associated with posterior hemorrhage after multivariate adjustment (OR 2.66, 95\% CI 1.00-7.07, $\mathrm{p}=0.049$ ). Their topographical analysis also showed good correlation between bleeding points associated with positive choroidal anastomosis and the anatomical distribution of the choroidal arteries, including the thalamus and the wall of the atrium. ${ }^{3}$ It is still unclear why choroidal and thalamic anastomoses are more prone to hemorrhage in patients with MMD, and further studies are warranted to resolve this issue. These findings may be related to the finding that the incidence of rebleeding is significantly higher in patients with MMD and posterior bleeding than in patients with anterior bleeding because these arteries give rise to the fragile moyamoya vessels that provide collateral blood flow through the thalamus and periventricular zone. ${ }^{11}$

Taken together, this study strongly suggests that the anterior-to-posterior shift of collateral channels can universally be observed during disease progression and aging in patients with MMD. Furthermore, the anterior-toposterior shift of collateral channels may be closely related to the onset of hemorrhagic stroke in adult patients with MMD because the abnormal dilation and extension of the PCoA, AChA, and PChA is directly linked to the occurrence of hemorrhagic events. ${ }^{3}$ The novel concept of the anterior-to-posterior shift of the collateral channels in patients with MMD may be useful for discussing the underlying mechanisms of hemorrhagic stroke in adult patients and for establishing more effective strategies that prevent the first-ever onset and recurrence of hemorrhagic stroke in patients with MMD.

Finally, there are some limitations in this study. The sample size of hemorrhagic-type hemispheres was too small $(n=11)$ to discuss the relationship between onset type and the development of collateral channels. However, the prevalence of hemorrhagic-type MMD is not so high, ${ }^{1}$ and thus further cooperative studies with larger cohorts are warranted. In addition, it should also be noted that the interobserver or intraobserver variabilities for grading collateral channels were not evaluated in this study. These factors should be evaluated in a future multicenter cohort study. 


\section{Conclusions}

This study strongly suggests that the collateral channels through moyamoya vessels longitudinally shift from the anterior to posterior component during disease progression and patient aging, which may be closely related to the onset of hemorrhagic stroke in adult patients with MMD. This novel concept of an anterior-to-posterior shift in the collateral channels would help elucidate the underlying mechanisms of hemorrhagic stroke in adult patients with MMD and help develop novel strategies that prevent hemorrhagic stroke in patients with MMD, although further studies with larger cohorts are warranted.

\section{Acknowledgments}

This study was supported by a grant (no. H26-078 awarded to S.K.) from the Research Committee on Moyamoya Disease, which is sponsored by the Ministry of Health, Labor, and Welfare of Japan.

\section{References}

1. Fukui M: Current state of study on moyamoya disease in Japan. Surg Neurol 47:138-143, 1997

2. Funaki T, Fushimi Y, Takahashi JC, Takagi Y, Araki Y, Yoshida K, et al: Visualization of periventricular collaterals in moyamoya disease with flow-sensitive black-blood magnetic resonance angiography: preliminary experience. Neurol Med Chir (Tokyo) 55:204-209, 2015

3. Funaki T, Takahashi JC, Houkin K, Kuroda S, Takeuchi S, Fujimura M, et al: Angiographic features of hemorrhagic moyamoya disease with high recurrence risk: a supplementary analysis of the Japan Adult Moyamoya Trial. J Neurosurg 128:777-784, 2018

4. Funaki T, Takahashi JC, Yoshida K, Takagi Y, Fushimi Y, Kikuchi T, et al: Periventricular anastomosis in moyamoya disease: detecting fragile collateral vessels with MR angiography. J Neurosurg 124:1766-1772, 2016

5. Irikura K, Miyasaka Y, Kurata A, Tanaka R, Fujii K, Yada K, et al: A source of haemorrhage in adult patients with moyamoya disease: the significance of tributaries from the choroidal artery. Acta Neurochir (Wien) 138:1282-1286, 1996
6. Kuroda S, Houkin K: Moyamoya disease: current concepts and future perspectives. Lancet Neurol 7:1056-1066, 2008

7. Liu W, Zhu S, Wang X, Yue X, Zhou Z, Wang H, et al: Evaluation of angiographic changes of the anterior choroidal and posterior communicating arteries for predicting cerebrovascular lesions in adult moyamoya disease. J Clin Neurosci 18:374-378, 2011

8. Morioka M, Hamada J, Kawano T, Todaka T, Yano S, Kai $\mathrm{Y}$, et al: Angiographic dilatation and branch extension of the anterior choroidal and posterior communicating arteries are predictors of hemorrhage in adult moyamoya patients. Stroke 34:90-95, 2003

9. Research Committee on the Pathology and Treatment of Spontaneous Occlusion of the Circle of Willis: Guidelines for diagnosis and treatment of moyamoya disease (spontaneous occlusion of the circle of Willis). Neurol Med Chir (Tokyo) 52:245-266, 2012

10. Suzuki J, Takaku A: Cerebrovascular "moyamoya" disease. Disease showing abnormal net-like vessels in base of brain. Arch Neurol 20:288-299, 1969

11. Takahashi JC, Funaki T, Houkin K, Inoue T, Ogasawara K, Nakagawara J, et al: Significance of the hemorrhagic site for recurrent bleeding: prespecified analysis in the Japan Adult Moyamoya Trial. Stroke 47:37-43, 2016

\section{Disclosures}

The authors report no conflict of interest concerning the materials or methods used in this study or the findings specified in this paper.

\section{Author Contributions}

Conception and design: Yamamoto, Kuroda. Acquisition of data: Yamamoto. Analysis and interpretation of data: Yamamoto. Drafting the article: Yamamoto. Critically revising the article: all authors. Reviewed submitted version of manuscript: Hori, Kashiwazaki, Akioka, Kuwayama, Kuroda. Statistical analysis: Yamamoto. Study supervision: Kuroda.

\section{Correspondence}

Shusuke Yamamoto: University of Toyama, Japan. s.yamamoto1007@gmail.com. 